\title{
Nosocomial Infections in Pediatric Intensive Care Units
}

\author{
Rakesh Lodha, Uma Chandra, Mouli Natchu, Mrinal Nanda and S.K. Kabra \\ Department of Pediatrics, All India Institute of Medical Sciences, Ansari Nagar, New Delhi, India
}

\begin{abstract}
Nosocomial infections are a significant problem in pediatric intensive care units. While Indian estimates are not available, western PICUs report incidence of 6-8\%. The common nosocomial infections in PICU are bloodstream infections (20-30\% of all infections), lower respiratory tract infections (20-35\%), and urinary tract infections (15-20\%); there may be some differences in their incidence in different PICUs. The risk of nosocomial infections depends on the host characteristics, the number of interventions, invasive procedures, asepsis of techniques, the duration of stay in the PICU and inappropriate use of antimicrobials. Most often the child had endogenous flora, which may be altered because of hospitalization, are responsible for the infections. The common pathogens involved are Staphylococcus aureus, coagulase negative staphylococci, E. coli Pseudomonas aeruginosa, Klebsiella, enterococci, and candida. Nosocomial pneumonias predominantly occur in mechanically ventilated children. There is no consensus on the optimal approach for their diagnosis. Bloodstream infections are usually attributable to the use of central venous lines; use of TPN and use of femoral site for insertion increase the risk. Urinary tract infections occur mostly after catheterization and can lead to secondary bacteremia. The diagnostic criteria have been discussed in the review. With proper preventive strategies, the nosocomial infection rates can be reduced by up to $50 \%$; handwashing, judicious use of interventions, and proper asepsis during procedures remain the most important practices. [Indian J Pediatr 2001; 68 (11) : 1063-1070]
\end{abstract}

\section{Key words : Nosocomial infections; Intensive care; Children; Prevention}

Nosocomial or hospital acquired infections are all infections that occur during a patient's hospitalization and not present or incubating at admission. Also any infection that appears to have been acquired in hospital but does not manifest until after discharge is judged to be a nosocomial infection. Therefore, all infections diagnosed 48 hours after admission till 72 hours after discharge should be considered as nosocomial. ${ }^{1,2}$

Nosocomial infections are a significant problem in pediatric intensive care units. The National Nosocomial Infection Surveillance System (NNIS) in the United States reports a rate of 14.1 nosocomial infections per 1000 patient days for pediatric ICUs. ${ }^{3}$ The overall nosocomial infection rates in the PICUs in the west are reported to be 6- $8 \% .^{3-5}$ These rates are lower than those seen in adult intensive care units., ${ }^{3,6}$ The infections in pediatric intensive care units (PICU) are more widespread than in general pediatric wards. The infection rates depend on the type of PICU: multidisciplinary, medical, surgical, or cardiac. There are no data on the incidence of nosocomial infections in Indian PICUs. It is possible that infection rates here are higher than those reported in the west.

According to NNIS, primary bloodstream infections are the commonest nosocomial infections $(28 \%)$ in PICUs followed by the lower respiratory tract infections (21\%) and urinary tract infection (UTI) $(15 \%){ }^{3}$ In another study, lower respiratory tract infections were the commonest

Reprint requests : Dr. Rakesh Lodha, Assistant Professor, Department of Pediatrics, All India Institute of Medical Sciences, Ansari Nagar, New Delhi- 110029. E-mail : skkabra@hotmail.com
(35\%), followed by bloodstream infections (21\%) and UTI $(16 \%) .{ }^{4}$ These variations may arise from the differences in the proportion in the children ventilated in the PICUs. In adults, UTI is the commonest nosocomial infection followed by surgical infection and lower respiratory tract infections. ${ }^{6}$ Staphylococcous aureus, coagulase negative staphylococci, E. coli, Pseudomonas aeruginosa, Klebseilla, enterococci and Candida are the significant pathogens in pediatric services in the various sites examined., ${ }^{3,4}$

The risk of nosocomial infections in a PICU is the direct consequence of the severity of illness, ${ }^{4}$ the level of invasive monitoring, the indiscriminate use of antimicrobials and the nature of diagnostic procedures. The NNIS data emphasizes that the duration of stay in an ICU is an important determinant of nosocomial infection; therefore, the length of stay should be one of the denominators in calculation of nosocomial infection rates. ${ }^{3}$ In addition, it highlight the importance of invasive devices (endotracheal tubes, intravascular catheters, urinary catheters) in development of nosocomial infections. ${ }^{3}$ In order to control for device usage and duration the infection rates can be represented as ratios per thousand device days. According to the latest report, ventilator associated pneumonia (VAP) occurrence is 5.7 episodes per thousand ventilator days, UTI 5.2 per thousand catheter days, and bloodstream infection is 8 per thousand central line days. ${ }^{3}$ In the same study, it was observed that $91 \%$ of all nosocomial bloodstream infections occurred in children with central venous lines, $95 \%$ of nosocomial pneumonias occurred in those on 


\section{Rakesh Lodha et al}

mechanical ventilation and $77 \%$ of UTIs in children with urinary catheters. ${ }^{3}$ These figures highlight the important role of various devices in nosocomial infections.

Nosocomial infections may be caused by organisms that originate from exogenous sources in hospital or from endogenous sources such as child's own flora. Because of alteration of child's flora associated with the illness and hospitalization, such distinction may not be easy. A recent study has shown that the mean number of days after admission to PICU to abnormal colonization were as follows: gastric aspirate, 2 days; tracheal aspirate, 5 days; urine 10 days; and stool, 4 days. The altered flora mainly included gram-negative bacilli and Staphylococcus aureus, which were often antibiotic resistant. ${ }^{7}$

The hospital environment contributes minimally to acquisition and spread of most endemic nosocomial infections directly. Even if environmental surfaces are contaminated with microbes, they can be spread to patients by hand contact only ${ }^{8}$. Occasionally, environment may be responsible for life threatening nosocomial infections such as aspergillus in immunocompromised individuals.

It is estimated that the overall mortality attributed to nosocomial infections is about $10 \% .{ }^{9}$ The mortality is associated with nosocomial infections is multifactorial; some of these factors are the type of patient, the number of altered organs, and the microbes responsible for the infection. Blood stream infections caused by Klebsiella pneumoniae or the fungi have mortality range of 18$20 \% .10,11$ The nosocomial infections increase the duration of stay in hospital and also the cost of therapy.

We restrict further discussion to nosocomial pneumonias, bloodstream infection and urinary tract infections.

\section{NOSOCOMIAL PNEUMONIAS}

Pneumonias constitute a common but potentially life threatening complication of hospitalization. The mortality rates associated with nosocomial pneumonia ranges from $20-50 \%{ }^{12,13}$ Other studies have reported lower mortality rates. ${ }^{14}$

While a few pneumonias represent hematogenous seeding of the lungs from a distant suppurative focus; in most patients, subclinical aspiration of oropharyngeal secretions containing bacteria that have colonized the upper airway of the patient is responsible for nosocomial pneumonia. This flora includes both gram-positive and gramnegative bacteria, which the patient acquires within 1-4 days after hospitalization. The risk factors for upper airway colonization includc acidosis, hypotension, endotracheal intubation, and broad-spectrum antimicrobial therapy. ${ }^{15}$ Only a few patients will develop nosocomial pneumonia in absence of colonization of the upper airways. ${ }^{13}$ Most nosocomial pneumonias are caused by gram-negative organisms. ${ }^{3,14}$ Bacteria of enterobacteriaceae family eg. E. coli, Klebsiella are usually isolated from the hypophar- ynx and rectum before they are isolated from the trachea. ${ }^{12}$ However, non-Enterobacteriaceae e.g. P. aerugino$s a$, Acinetobacter are rarely demonstrated prior to their isolation from the trachea. ${ }^{12}$ This suggests that colonization with enterobacteriaceae occurs from patient's endogenous flora and that non-Enterobacteriaceae bacteria have environmental origin. Therefore, the hands of the health care workers and components of the respiratory therapy equipment may be important factors in the transmission of bacteria.

The most important risk factor for nosocomial pneumonia in children is endotracheal intubation. Nosocomial pneumonias are nearly four times more common in intubated patients than in non-intubated patients. ${ }^{12,14}$ The risk may be greater with tracheostomy tubes. The filtration system of upper airways and mucociliary system of the large airways is bypassed during intubation, predisposing the patient to colonization with potential pathogens. Children with asymptomatic or symptomatic aspiration are at risk. ${ }^{12}$ Children with tracheoesophageal fistula, pharyngeal aspiration, gastroesophageal reflux, pulmonary disease, malnutrition or immunodeficiency are at greater risk. The etiologic microorganisms for nosocomial pneumonia vary from hospital to hospital. The pediatrician must be familiar with the common microbes and the antimicrobial susceptibility of these microbes at his/ her hospital. Gram-negative bacilli are the most important cause; 3 ,15 staphylococci are the most important gram-positive organisms. Viruses such as respiratory syncytial virus are also important pathogens responsible for nosocomial pneumonia in PICUs. ${ }^{3}$ However, some authors have suggested that viruses are less frequent as nosocomial pathogens in PICUs than in other areas of pediatric hospitals. $^{16}$

The optimal approach for diagnosing nosocomial pneumonia remains elusive. Clinical features, chest radiographs and culture of respiratory secretions have been used to establish the diagnosis. However, this approach is likely to overestimate the diagnosis. Fever and leukocytosis are also non-specific. Cough and sputum production are infrequently diagnostic of pneumonia in intubated children. Purulent tracheal secretions may be due to tracheitis, tracheobronchitis or pneumonia; differentiation can be difficult. Conditions such as atelectasis, pulmonary edema, pulmonary hemorrhage and acute respiratory distress syndrome (ARDS) may be confused with pneumonia. ${ }^{16}$ Therefore, clinical suspicion has to be strong for diagnosing nosocomial pneumonia. A change in the child's status such as desaturation, increased requirement for supplemental oxygen, increase in ventilator settings or fever that are not explained by other events can be helpful in the diagnosis. In view of these problems and controversies Centers for Disease Control and Prevention have provided clinicians with guidelines for diagnosis of nosocomial LRTI. ${ }^{1,2}$ 
Once nosocomial pneumonia is suspected attempts should be made to identify the etiologic agent. Qualitative cultures obtained from endotracheal tubes do not predict the causative agent of lower respiratory tract infections. Quantitative cultures appear to be superior to qualitative cultures. ${ }^{18}$ Blood cultures are usually negative. Transtracheal aspiration in non-intubated patients, percutaneous thin needle lung aspirations, bronchoalveolar lavage (bronchoscopic or blind), and protected bronchoscopic samples of the lower airways have all been suggested as methods for diagnosis, where contamination of lower respiratory secretions with upper airway flora is prevented. ${ }^{19,20}$ In the absence of gold standard criteria for the diagnosis of ventilator-associated pneumonia, quantitative cultures and microscopic examination of the lower respiratory tract secretions are the diagnostic tests of choice. This can provide accurate diagnosis and identification of causative organism. These can also help in diagnosis of ventilator-associated pneumonia in children with ARDS. ${ }^{21}$

\section{Prevention of Nosocomial Pneumonias}

The colonization of upper airways by pathogenic microbes and thereby, the risk of nosocomial pneumonia can be reduced by several measures. Effective hand washing by the health care personnel can reduce the risk of nosocomial pneumonias. ${ }^{22}$ Various chemicals such as chlorhexidine or rubs containing alcohol may be used. The reduction in the number of microorganisms on hands is related to the volume and number of times they are used. ${ }^{23}$ In addition, the hospital workers should comply with hospital infection control policies.

The gastrointestinal tract is an important source for endogenous upper airway colonization. Use of antacids and $\mathrm{H} 2$ blockers raise gastric $\mathrm{pH}$ and facilitate gastric microbial colonization. When indicated, instead of $\mathrm{H} 2$ blockers and antacids, sucralfate may be used for prophylaxis against gastric bleeding as the gastric $\mathrm{pH}$ remains low with its use. ${ }^{24}$ The use of selective decontamination of the gut using antimicrobial such as tobramycin, gentamycin, polymyxin and nystatin is controversial and is not recommended. ${ }^{25}$

Contaminated respiratory therapy equipments have been implicated in nosocomial pneumonias. ${ }^{25}$ Resuscitation bags, ventilator tubings, nebulizers should be disinfected. Only sterile fluids should be nebulized or used in humidifiers. Personnel taking care of intubated children should wash their hands before and after delivering care. The ventilator circuit tubings should be changed no more often than every 48 hours. ${ }^{17,25}$ Care should be taken to prevent contamination during suctioning; endotracheal suctioning should be performed as needed to remove secretions. ${ }^{26}$ Positioning of patients with head end elevation does reduce the risk of aspiration and nosocomial pneumonia. ${ }^{27}$

\section{Other Nosocomial Respiratory Tract Infections}

Bacterial tracheitis usually is secondary to a primary viral upper respiratory tract infection, most often parainfluenza virus. ${ }^{17}$ The data on nosocomial bacterial tracheitis is scant. ${ }^{14}$ Most nosocomial upper respiratory tract infections are viral and appear 2 weeks after admission. ${ }^{28}$ Bacteria are implicated in sinusitis and otitis media. Sinusitis may occur in a significant number of patients who have undergone nasotracheal intubation. ${ }^{29}$ Nasogastric tubes also predispose to sinusitis. The diagnosis usually has to be confirmed by radiology.

\section{BLOODSTREAM INFECTIONS}

While intravascular catheters provide lifesaving therapy, they also provide a route for microorganisms to bypass normal host defenses and can cause serious infections. Primary bacteremia is defined as a bloodstream infection occurring in a patient with no evidence of localized infection while secondary bacteremia is defined as a bloodstream infection with evidence of infection at another site that is the source of the bloodstream infection. More than $90 \%$ of all nosocomial bloodstream infections are in children with central venous lines. ${ }^{3}$

Infections may occur due to microbes from the skin moving along the catheter surface where catheter enters the skin or due to microbes gaining access to the catheter through the catheter hub and moving down the endoluminal surface of the catheter to the bloodstream. ${ }^{30,31}$ The former mechanism is more often seen in short-term catheters and the latter in long-term catheters. Formation of biofilms over the implanted devices also may have a role in occurrences of the infections.

The most common organisms in bloodstream infections are coagulase negative staphylococcus, Staphylococcus aureus and enterococci. ${ }^{3}$ Among the gramnegative bacteria Enterobacter, Pseudomonas aeruginosa, Klebsiella pneumoniae, and E. coli are the most important organisms. ${ }^{3}$ In immunocompromised children and those who have received a variety of broad spectrum antibacterials, fungal infections are more frequent.

The most important risk factor for catheter related bloodstream infections is the type of catheter used. Central venous catheters account for about $2 \%$ of all catheters inserted and more than $90 \%$ of all catheterrelated bloodstream infections. ${ }^{3}$ The risk is probably greater with multilumen central venous catheters. ${ }^{32}$ Sub optimal care of central venous catheters especially when used for total parenteral nutrition (TPN) is another risk factor. Lipid infusions have specifically been associated with risk of catheter-related bloodstream infections due to coagulase negative staphylococcus. ${ }^{33}$ The risk of infection increases linearly with the duration of catheterization. The site of insertion is an important determinant of the risk of infection; rates for femoral catheters are higher than that for jugular or subclavian catheters. ${ }^{34}$ Peripherally inserted central catheters (PICC lines) may have lower infection rates. Inadvertent contamination during insertion may be an important risk factor for infection. This is highlighted by an inverse relation between the 


\section{Rakesh Lodha et al}

total number of catheters inserted by the physician inserting the catheter and the risk of significant catheter colonization..$^{35}$ Improper management of catheter after insertion significantly increases the risk of infection. Transparent dressings may be associated with a higher rate of blood stream infections. ${ }^{36}$

Local catheter-related infections usually manifests with local inflammation erythema, tenderness and/or purulent discharge from the catheter tract.

The presentation of bloodstream infections may be either acute or insidious. There may be signs of sudden deterioration, elevated body temperature, chills and tachycardia or there may be intermittent fever and failure to improve from their basic illness. Whenever catheter related bacteremia is suspected, blood cultures should be obtained from peripheral blood as well as all catheter sites to correlate and differentiate colonization from infection. Catheter related bloodstream infection is defined as the isolation of same organism from blood cultures that is shown to be significantly colonizing the catheter of a patient with clinical features of bloodstream infection in the absence of any other local infection caused by the same organism that could give rise to bloodstream infection. ${ }^{1,2}$

\section{Prevention of Bloodstream Infections}

The major factors associated with development of catheter-related nosocomial infections are : (i) the sterility of the technique of insertion and maintenance of the catheter throughout its life, (ii) type of solution being administered through the intravenous line, (iii) number of "break ins" into the catheters system and intravenous tubing, (iv) the presence of infection elsewhere in the body. ${ }^{37}$ The following measures may help in reducing catheter-related infections :

a) Selection of subclavian, basilic or cephalic vein site rather then femoral or internal jugular vein.

b) Using maximal aseptic technique for catheter "insertion"38

c) Mupirocin ointment may reduce the risk of bacterial colonization of catheters but may increase colonization rate of fungi.

d) Using cotton gauze rather than transparent dressing. ${ }^{36}$

e) Having an experienced physician insert the catheter.

f) Avoid use of TPN catheters for other than infusion of TPN.

f) Have adequate staff for management of patients with central venous catheters.

There have been some technologic advances to prevent catheter- related bloodstream infections. These include a short-term chlorhexidine- silver sulfadiazine-impregnated catheters, minoycline- rifampicin- impregnated catheters, a catheter hub containing an iodinated alcohol solution, and chlorhexidine-impregnated sponge dressings. ${ }^{39}$

\section{NOSOCOMIAL URINARY TRACT INFECTIONS}

Urinary tract infections are common nosocomial infections in pediatric practice. ${ }^{40}$ Pediatric patients have a cumulative risk of UTI of $30 \%$ when catheterized ${ }^{40}$ Risk figures seem to be higher in infants but still compare better than with the cumulative risk observed in catheterized adults. Catheter associated UTI (CAUTI) rank second after intravascular catheter related bloodstream infections as a cause of secondary bacteremia. ${ }^{41}$ CAUTIs may extend hospital stay, ${ }^{42}$ increase cost of therapy ${ }^{43}$ and often lead to unwarranted antimicrobial therapy especially when the UTIs are asymptomatic. CAUTIs have been shown to significantly increase hospital death rates in adults independent of the occurrence of sepsis of urogenital origin. ${ }^{44}$ However, a similar effect has not been seen in the pediatric age group. ${ }^{45}$ The urinary tract can be an important hospital reservoir of nosocomial antimicrobial-resistant organisms ranging from resistant enterobacteriaceae, pseudomonas, staphylococci and candida.

Largely, the origin of the organisms causing the UTI can be traced either to the patient's own large bowel, perineum or to the hands of care providers. Contamination of the urinary tract by microbes occurs either through the catheter lumen or by ascending up along the catheter during or after insertion ${ }^{46}$ Extraluminal contamination is the commonest; it can occur by direct inoculation when the catheter is inserted or later, by the ascent of microbes from the perineum by capillary action in the thin mucus film contiguous to the external catheter surface. ${ }^{47}$ Intraluminal contamination occurs by reflux of microorganisms gaining access to the catheter lumen because of failure of closed drainage or contamination of urine in the collection bag. Pyelonephritis from bloodstream infections are rare but may occur in newborns and infants.

The most important risk factor for nosocomial UTI is catheterization. The timing, purpose and duration of catheterization are also important. Malnutrition, renal dysfunction, urological procedures and stenting, open drainage, diarrhea, periurethral skin contamination also play a role in nosocomial UTIs.

The criteria for the diagnosis of an UTI would depend on the catheterization status as follows. Some uncertainty still exists in the diagnosis in catheterized children. The catheter, by simple irritation to the bladder, may cause pyuria; the drainage provided by the catheter may mask the clinical symptoms, and in some patients, the underlying disease process may cause fever or render the patient incapable of indicating the symptoms. The following are the guidelines for diagnosis of UTI

- Clean catch mid stream urine growing $>10^{5}$ bacteria/ml of urine. ${ }^{48}$

- Any growth in a suprapubic sample (gold standard for confirmation of diagnosis). 


\section{Nosocomial Infections in Pediatric Intensive Care Units}

- Bacterial counts of $>10^{2} / \mathrm{ml}$ in samples aspirated with a needle from the catheter in those patients with indwelling catheters. ${ }^{49}$

\section{Prevention of Nosocomial Urinary Tract Infections}

A multitude of practices have been recommended and used to prevent the occurrence of UTIs. Controlled trials are yet to prove the efficacy of some of these. The catheterizations should be kept to a minimum. The need for catheterization must be strictly evaluated and catheterization must be replaced by a closed condom drainage whenever possible. ${ }^{50}$ Suprapubic catheterization may be linked to a lesser risk of a CAUTI and may be more comfortable to some patients. ${ }^{50}$ When thought to have fulfilled their need the catheters must be immediately removed. ${ }^{51}$ Strict asepsis should be maintained during insertion of the catheter using sterile gloves, drapes, and local antiseptics.

Closed drainage must be strictly maintained and this has been shown to bring down the rates of infection to less than $25 \%$ for up to 2 weeks of catheterization. ${ }^{52}$ The closed drainage must be maintained with the collection tubing and bag below the level of the patient's bladder and the tubing must always be above the level of the bag. Other positions, by allowing backflow of urine, increase the risk of infection by two-fold. ${ }^{53}$ When in place the closed drainage system must be handled and manipulated as infrequently as possible. Antibiotic prophylaxis does reduce the frequency of infections but is not universally recommended as it selects multidrug resistant strains when the infection occurs.

Antiinfective lubricants, sealed catheter tubing junctions, antireflux valves, bladder or bag irrigation with antiinfective solution, antibiotic, silver hydrogel impregnated catheter materials are the newer techniques being studied for their role in reducing the risk of nosocomial UTIs. ${ }^{46}$

\section{STRATEGIES TO REDUCE THE INCIDENCE OF NOSOCOMIAL INFECTIONS}

For reducing the incidence of nosocomial infection, each PICU should have an infection control program. There should be a written description of the goals, objectives, and structure of program. A team of health professionals should ensure implementation of the policies and compliance on the part of the PICU team. Well-directed infection control activities can reduce the nosocomial infection rates by up to $50 \% .{ }^{54}$

The importance of hand washing and hand disinfection is well understood. The appropriate hand washing technique includes wetting the hands, taking soap, rubbing hands to produce a lather, and performing wash movements that include rubbing palm to palm, right palm over left dorsum and vice versa, palm to palm with fingers interlaced, backs of fingers to opposing palm with fingers interlocked, rotational rubbing of right thumb clasped in left palm and vice versa, rotational rubbing with clasped fingers of right hand in palm of left hand and with changed roles. ${ }^{55}$ The whole procedure should not take less than 30 seconds. After washing, hands should be dried with disposable paper or cloth towel. It has been noticed that health personnel practice hand washing in only $25-50 \%$ of the opportunities. ${ }^{56}$ In order to improve compliance, various hygienic hand rubs can be used. Rubbing of 3- $5 \mathrm{ml}$ of a fast acting antiseptic preparation on to both hands can be an effective substitute to hand washing. The various preparations available include n-propanol, isopropanol, ethanol, and chlorhexidine diacetate..$^{55}$

In addition to the specific measures mentioned earlier for prevention of specific nosocomial infections, proper sterilization/disinfection of various medical items is mandatory. Aseptic precautions should be followed strictly whenever any invasive procedure is being carried out.

A well-nourished child is less likely to acquire nosocomial infection than a malnourished one. Even in PICU, nutrition should be given due attention. Enteral nutrition appears to be better than parenteral nutrition. Enteral nutrition may have a favorable impact on gastrointestinal immunologic function and infectious morbidity. ${ }^{57}$ There has been considerable interest in the role of immune-enhancing enteral diets (containing glutamine, arginine, mRNA, omega- 3 fatty acids from fish oil) in reduction of nosocomial infections and mortality in ICUs. ${ }^{58,59}$

Appropriate and rational prescription of antibiotics is essential to prevent emergence of resistant strains. ${ }^{56}$ There should be constant surveillance and periodic review of the antibiotic policies and prescriptions. With careful microbiologic monitoring, cycling of antibiotics for empiric therapy can help in reducing the emergence of drug resistance. ${ }^{56}$

Adequate and well trained staff- both nursing staff and physicians- are essential for infection control. Various studies have demonstrated the adverse effects of understaffing on nosocomial infection rates. ${ }^{60,61}$ Education of the staff about various infection control practices and procedure-specific guidelines has an important role in the reduction of incidence of nosocomial infections. The education program should be on a continuing basis with periodic evaluation of the knowledge and practices.

Surveillance of nosocomial infections is an essential element of any infection control program. The most important goal of surveillance is to reduce the risk of acquiring nosocomial infections. This provides data useful for identifying infected patients, determining the site of infections and identifying the factors that contribute to nosocomial infections. Use of uniform definitions is critical for proper collection of data and inter-hospital comparisons. Standard CDC definitions that include laboratory and clinical criteria may be used. ${ }^{1,2}$ The major purpose of surveillance is to establish baseline infection rates and identifying outbreaks. This data can be used 


\section{Rakesh Lodha et al}

effectively to convince health care workers to accept recommended preventive practices. Control measures can be evaluated objectively if the surveillance is good.

\section{REFERENCES}

1. Garner JS, Jarvis WR, Emori TG, Haran TC, Hughes JM. CDC definitions for nosocomial infections, 1988. J Infect Control $1988 ; 16: 128-40$.

2. Gaynes RP, Horan TC. Surveillance of nosocomial infections. In Mayhall CG, ed. Hospital Epiderniology and Infection Control, 2nd edn. Lippincott Williams \& Wilkins, Philadelphia. 1999: 1296-1307.

3. Richards MJ, Edwards JR, Culver DH, Gaynes RP. The National Nosocomial Infection Surveillance System. Nosocomial infections in pediatric intensive care units in the United States. Pediatrics 1999; $103: 39$.

4. Singh-Naz N, Sprague BM, Patel KM, Pollack MM. Risk factors for nosocomial infection in critically ill children: A prospective cohort study. Crit Care Med 1996; 24 : 875-878.

5. Brown RB, Stechenberg B, Sands M, Hosmer D, Ryczak M. Infections in a pediatric intensive care unit. Am J Dis Child $1987 ; 141: 267-270$.

6. Richards MJ, Edwards JE, Culver DH, Gaynes RP Nosocomial infections in medical ICUs in the United States. Crit Care Med 1999; 27 : 887-892.

7. Bowen-Jones J. Nosocomial colonization and infection in a pediatric respiratory intensive care unit. S Afr Med J 1992; 82: 309-313.

8. Vesley D, Streifel AJ. Environmental services. In Mayhall CG, ed. Hospital Epidemiology and Infection Control. 2nd edn. Lappincott Coillianis \& Wilkins, Philadelphia. 1999 : 10471053.

9. Millikan J, Tait GA, Ford-Jones EL et al. Nosocomial infections in a pediatric intensive care unit. Crit Care Med 1988; $16: 267$ 270 .

10. Bonadio WA. Klebsiella pneumoniae bacteremia in children. Am J Dis Child 1989; 143: 1061-1063.

11. Turner RB, Donowitz LG, Hendley JO. Consequences of candidemia for pediatric patients. Am J Dis Child 1985; 139: 178-180.

12. Jacobs RF. Nosocomial infection in children. Infection 1991; 19 : 64-72.

13. Craven DE, Kunches LM, Kilinsky V, Lichtenberg DA, Make BJ, McCabe WR. Risk factors for pneumonia and fatality in patients receiving continuous mechanical ventilation. Am Rev Respir Dis 1986; 133 : 792-796.

14. Fayon MJ, Tucci M, Lacroix J et al. Nosocomial pneumonia and tracheitis in a pediatric intensive care unit: a prospective study. Am J Respir Crit Care Med 1997; 155 : 162-169.

15. Schwartz SN, Dowling JN, Benkovic C et al. Sources of gram negative bacilli colonizing the trachea of intubated patients. J Infect Dis 1978; 138 : 227-231.

16. Ford-Jones EL, Mindolff CM, Langley JM et al. Epidemiologic study of 4684 hospital acquired infections in pediatric patients. Pediatr Infect Dis J 1989; 8 : 668-675.

17. Schutze GE, Yamauchi T. Nosocomial bacterial infections of the central nervous system, upper and lower respiratory tracts, and skin in pediatric patients. In Mayhall CG, ed. Hospital Epidemiology and Infection Control, 2nd edn. Lippincott Williams \& Wilkins, Philadelphia. 1999: 615-627.

18. Torres A, Martos A, de La Bellacasa JP et al. Specificity of endotracheal aspiration, protected specimen brush, and bronchoalveolar lavage in mechanically ventilated patients. Am Rev Respir Dis 1993; 147 : 952-957.

19. Marquette $\mathrm{CH}$, Georges $\mathrm{H}$, Wallet $\mathrm{F}$. Diagnostic efficiency of endotracheal aspirates with quantitative bacterial cultures in intubated patients with suspected pneumonia: comparison with protected specimen brush. Am Rev Respir Dis 1993; 148 : 138-144.

20. Labenne M, Poyart C, Rambaud C et al, Blind protected specimen brush and bronchoalveolar lavage in ventilated children. Crit Care Med 1999; 27: 2537-2543.

21. Mayhall CG. Ventilator-associated pneumonia or not? Contemporary diagnosis. Emerg Infect Dis 2001; 7 : 200-204.

22. Kollef MH. The prevention of ventilator associated pneumonia. $N$ Engl J Med 1999; $340: 627-633$.

23. Larson E. A causal link between handwashing and risk of infection? Examination of evidence. Infect Control Hosp Epidemiol 1998; 9 : 28-36.

24. Driks MR, Craven DE, Celli BR et al. Nosocomial pneumonia in intubated patients given sucralfate as compared with antacids or histamine type 2 blockers: the role of gastric colonization. N Engl J Med 1987; 317 : 1376-1382.

25. Centers for Disease Control and Prevention. Guidelines for prevention of nosocomial pneumonia. MMWR 1997; 46 (RR1) : 1-79.

26. Nelson $S$, Chidiac C, Summer WR. New strategies for preventing nosocomial pneumonia: which common interventions leave patients at increased risk? J Crit Illness $1988 ; 3: 12-24$.

27. Drakulovic MB, Torres A, Bauer TT, Nicolas JM, Nogue $S$, Ferrer M. Supine body position as a risk factor for nosocomial pneumonia in mechanically ventilated patients: a randomized trial. Lancet 1999; 354 : 1835-1836.

28. Jarvis WR. Epidemiology of nosocomial infections in pediatric patients. Pediatr Infect Dis J 1987; 6 : 344-351.

29. Hansen M, Poulsen MR, Bendixen DK et al. Incidence of sinusitis in patients with nasotracheal intubation. $\mathrm{Br} J$ Anaesth $1988 ; 61: 231-2$.

30. Cheesbrough JS, Finch RG, Burden RP. Aprospective study of the mechanisms of infection associated with hemodialysis catheters. J Infect Dis 1986; 154: 579-589.

31. Moro ML, Vigano EF, Lepri AC. Risk factors for central venous catheter related infections in surgical and intensive care units. Infect Control Hosp Epidemiol 1994; 15 : 253-264.

32. Yeung C, May J, Hughes R. Infection rate for single lumen vs triple lumen subclavian catheters. Infect Control Hosp Epidemiol 1998; 9: 154-8.

33. Shiro H, Muller E, Takeda $\mathrm{S}$ et al. Potentiation of Staphylococcus epidermidis catheter-related bacteremia by lipid infusions. J Infect Dis 1995; 171 : 220-224.

34. Richet $H, H u b e r t ~ B, N i t e m b e r g ~ G$ et al. Prospective multicenter study of vascular catheter- related complications and risk factors for positive patients. J Clin Microbiol 1990; 28: 2520-2525.

35. Armstrong CW, Mayhall CG, Miller KB et al. Prospective study of catheter replacement and other risk factors of infection of hyperalimentation catheters. J Infect Dis 1986; 154: 808-816.

36. Conly JM, Grieves K, Peters B. A prospective, randomized study comparing transparent and dry gauze dressings for central venous catheters. J Infect Dis 1989; 159:310-319.

37. Stein $\mathrm{F}$, Trevino $\mathrm{R}$. Nosocomial infections in the pediatric intensive care unit. Pediatr Clin North Am 1994; 41 : 1245-1257.

38. Raad I, Hohn DC, Gilbreath BJ et al. Prevention of central venous catheter related infections by using maximal sterile precautions during insertion. Infect Control Hosp Epidemiol 1994; 15 : 231-238.

39. Mermel LA. New technologies to prevent intravascular catheter-related bloodstream infections. Emerg Infect Dis 2001; 7 : 197-199.

40. Kasian GF. Bacteriologic surveillance of indwelling urinary catheters in pediatric intensive care unit patients. Crit Care 


\section{Nosocomial Infections in Pediatric Intensive Care Units}

Med 1988; $16: 679-682$.

41. Krieger JN, Kaiser DIL, Wenzel RP. Urinary tract etiology of bloodstream infections in hospitalized patients. I Infect Dis 1983; $148: 57-62$.

42. Givens CD, Wenzel RP. Catheter-associated urinary tract infections in surgical patients: a controlled study on the excess morbidity and costs. J Urol 1980; $24: 646-650$.

43. Patton JP, Nash DB, Abrutyn E. Urinary tract infection: economic considerations. Med Clin North Am 1991; 75 : 495513.

44. Platt R, Polk BF, Murdock B, Rosner B. Mortality associated with nosocomial urinary tract infection. N Engl J Med 1982; 307 : 637-641.

45. Lohr JA, Downs SM, Dudley S, Donowitz LG. Hospitalacquired urinary tract infections in pediatric patient: a prospective study. Pediatr Infect Dis J 1994; 13: 8-12.

46. Maki DG, Tambyah PA. Engineering out the risk for infection with urinary catheters. Emerg Infect Dis 2001; 7 : 342-347.

47. Tambyah PA, Halvorson K, Maki DG. A prospective study of the pathogenesis of catheter-associated with urinary tract infection. Mayo Clin Proc 1999; 74 : 131-136.

48. Kass EH. Asymptomatic infections of the urinary tract. Trans Assoc Am Physicians 1956; 69 : 56-60.

49. Stark RP, Maki DG. Bacteriuria in the catheterized patient. $N$ Engl J Med 1984; 311 : 560-564.

50. Warren JW. Urethral catheters, condom catheters, and nosocomial urinary tract infections. Infect Control Hosp Epidemiol 1996; 17 : 212-214.

51. Rabkin DG, Stifelman MD, Birkhoff J et al. Early catheter removal decreases incidence of urinary tract infections in renal transplant receipients. Transplant Proc 1998; $30: 4314-4316$.

52. Kunin CM, McCormack RC. Prevention of catheter-induced urinary tract infections by sterile closed drainage. $N$ Engl I Med $1966 ; 274: 1155-1161$.
53. Maki DG, Knasinski V, Tambyah PA. Risk factors for catheterassociated urinary tract infection: a prospective study showing the minimal effects of catheter care violations on the risk of CAUTI (Abstract). Infect Control Hosp Epidemiol 2000; $21: 165$.

54. Haley RW, Culver DH, White JW et al. The efficacy of infection surveillance and control programs in preventing nosocomial infections in US hospitals. Am J Epidemiol 1985; 121: 182-187.

55. Rotter ML. Hand washing and hand disinfection. In Mayhall CG, ed. Hospital Epidemiology and Infection Control, 2nd edn. Lippincott Williams \& Wilkins, Philadelphia. 1999; 1339-1355.

56. Weinstein RA. Controlling antimicrobial resistance in hospitals: infection control and use of antibiotics. Emerg Infect Dis 2001; 7 : 188-192.

57. Heyland DK, Cook DJ, Guyatt GH. Enteral nutrition in the critically ill patient: a critical review of the evidence. Intensive Care Med 1993; $19: 435-442$.

58. Galban C, Montigo JC, Mesejo A et al. An immune-enhancing enteral diet reduces mortality rate and episodes of bacteremia in septic intensive care patients. Crit Care Med 2000; 28 : 643648 .

59. Houdijk AP, Rijnsburger ER, Jansen J et al. Randomised trial of glutamine-enriched enteral nutrition on infectious morbidity in patients with multiple trauma. Lancet 1998; $352: 772-776$.

60. Fridkin SK, Pear SM, Williamson TH, Galgiani JN, Jarvis WR. The role of understaffing in central venous catheter-associated bloodstream infections. Infect Control Hosp Epidemiol 1996; 17 : 150-158.

61. Archibald LK, Manning ML, Bell LM, Banerjee $S$, Jarvis WR. Patient density, nurse-to-patient ratio and nosocomial infection risk in a pediatric cardiac intensive care unit. Pediatr Infect Dis J 1997; 16 : 1045-1048.

\section{APPENDIX \\ Nosocomial Infections : Definition of Infection Sites (modified from Ref 1, 2)}

INFECTION SITE : Pneumonia

DEFINITION : Pneumonia must meet at least one of the following criteria :

Criterion 1 : Patient has crepitations or dullness to percussion on the physical examination of the chest And At least one of the following:

a. new onset of purulent sputum or change in character of sputum

b. organisms cultured from blood

c. isolation of an etiologic agent from a specimen obtained by transtracheal aspirate, bronchial brushing, or biopsy.

Criterion 2 : Patient has a chest radiographic examination that shows new or progressive infiltrate, consolidation, cavitation, or pleural effusion

And

At least one of the following:

a. new onset of purulent sputum or change in character of sputum

b. organisms cultured from blood

c. isolation of an etiologic agent from a specimen obtained by transtracheal aspirate, bronchial brushing, or biopsy.

d. isolation of virus or detection of viral antigen in respiratory secretions

e. histopathologic evidence of pneumonia.
Criterion 3 : Patient $\leq 1$ year of age has at least two of the following signs or symptoms: apnea, bradycardia, wheezing, rhonchi, or cough And

At least one of the following :

a. increased production of respiratory secretions

b. new onset of purulent sputum or change in character of sputum

c. organisms cultured from blood

d. isolation of an etiologic agent from a specimen obtained by transtracheal aspirate, bronchial brushing, or biopsy.

e. isolation of virus or detection of viral antigen in respiratory secretions

f. histopathologic evidence of pneumonia

Criterion 4 : Patient $\leq 1$ year has a chest radiographic examination that shows new or progressive infiltrate, consolidation, cavitation, or pleural effusion

And

At least one of the following :

a. increased production of respiratory secretions

b. new onset of purulent sputum or change in character of sputum

c. organisms cultured from blood

d. isolation of an etiologic agent from a specimen obtained by transtracheal aspirate, bronchial 


\section{Rakesh Lodha et al}

brushing, or biopsy.

e. isolation of virus or detection of viral antigen in respiratory secretions

f. histopathologic evidence of pneumonia.

*Expectorated sputum cultures are not useful in diagnosis of pneumonia

*Findings from serial chest $x$-rays may be more useful than a single $x$-ray.

INFECTION SITE : Laboratory confirmed bloodstream infection DEFINITION : Laboratory-confirmed bloodstream infection must meet at least one of the following criteria:

Criterion 1 : Patient has a recognized pathogen cultured from one or more blood cultures

And

Organism cultured from blood is not related to an infection at another site.

Criterion 2 : Patient has at least one of the following signs or symptoms: fever $\left(>38^{\circ} \mathrm{C}\right)$, chills, or hypotension And

At least one of the following :

a. common skin contaminant is culture from two or more blood cultures drawn on separate occasions

b. common skin contaminant is cultured from at least one culture from a patient with an intravascular line, and the physician institutes appropriate antimicrobial therapy

c. positive antigen test on blood (e g. H. influenzae, $S$. pneumoniae)

and

signs and symptoms and positive laboratory results are not related to an infection at another site.

Criterion 3 : Patient $\leq 1$ year of age has at least one of the following signs or symptoms: fever $\left(>38^{\circ} \mathrm{C}\right)$, hypothermia $\left(<37^{\circ} \mathrm{C}\right)$, apnea, or bradycardia And

At least one of the following :

a. common skin contaminant is cultured from two or more blood cultures drawn on separate occasions

b. common skin contaminant is culture from at least one culture from a patient with an intravascular line, and the physician institutes appropriate antimicrobial therapy

c. positive antigen test on blood (e. g. H. influenzae, $S$. pneumoniae)

and

signs and symptoms and positive laboratory results are not related to an infection at another site.

INFECTION SITE : Clinical sepsis

DEFINITION : Clinical sepsis must meet at least one of the following criteria:

Criterion 1 : Patient has at least one of the following clinical signs or symptoms with no other recognized cause: fever $\left(>38^{\circ} \mathrm{C}\right)$, hypotension, or oliguria

And

Blood culture not done or no organisms or antigen detected in blood

And

No apparent infection at another site

And

Physician institutes treatment for sepsis.

Criterion 2 : Patient $\leq 1$ year of age has at least one of the following clinical signs or symptoms with no other recognized cause: fever $\left(>38^{\circ} \mathrm{C}\right)$, hypothermia $\left(<37^{\circ} \mathrm{C}\right)$, apnea, or bradycardia

And

Blood culture not done or no organisms or antigen detected in blood

And

No apparent infection at another site

And

Physician institutes treatment for sepsis.

INFECTION SITE : Symptomatic urinary tract infection

DEFINITION : A Symptomatic urinary tract infection must meet at least one of the following criteria:

Criterion 1 : Patient has at least one of the following sign or symptoms with no other recognized cause : fever $\left(>38^{\circ} \mathrm{C}\right)$, urgency, frequency, dysuria or suprapubic tenderness and

Patients has a positive urine culture that is $\geq 10^{5}$ microorganisms per $\mathrm{cm}^{3}$ of urine with no more than two species of microorganisms.

Criterion 2 : Patients has at least two of the following signs or symptoms with no other recognized cause: fever $\left(>38^{\circ} \mathrm{C}\right)$, Urgency, frequency, dysuria, or suprapubic tenderness and at least one of the following :

a. pyuria (urine specimen with $\geq 10 \mathrm{WBC} / \mathrm{mm}^{3}$ or $\geq 3$ WBC/high power field of unspun urine)

b. organism seen on Gram stain of unspun urine

c. at least two urine culture with repeated isolation of the same uropathogen (gram-negative bacteria or S.saprophyticus) with $\geq 10^{2}$ colonies $\mathrm{mL}$ in non voided specimens

d. $\leq 10^{5}$ colonies $/ \mathrm{mL}$ of a single uropathogen (gram negative bacteria or $\mathrm{S}$. saprophyticus) in a patient being treated with an effective antimicrobial agent for a urinary tract infection

e. physician diagnosis of a urinary tract infection

f. physician institutes appropriate therapy for a urinary tract infection

Criterion 3 : Patient $\leq 1$ year of age has at least one of the following signs or symptoms with no other recognized cause:

fever $\left(>38^{\circ} \mathrm{C}\right)$, hypothermia $\left(<37^{\circ} \mathrm{C}\right)$, apnea, bradycardia, dysuria, lethargy, or vomiting and patient has a positive urine culture, that is $\geq 10^{5}$ microorganisms per $\mathrm{cm}^{3}$ of urine with no more than two species of microorganisms.

Criterion 4 : Patient $\leq 1$ year of age has at least one of the following signs or symptoms with no other recognized cause: fever $\left(>38^{\circ} \mathrm{C}\right)$, hypothermia $\left(<37^{\circ} \mathrm{C}\right)$, apnea, bradycardia, dysuria, lethargy, or vomiting

And

At least one of the following:

a. pyuria (urine specimen with $\geq 10 \mathrm{WBC} / \mathrm{mm}^{3}$ or $\geq$ $3 \mathrm{WBC} /$ high power field of unspun urine)

b. organisms seen on Gram stain of unspun urine

c. atleast two urine culture with repeated isolation of the same uropathogen (gram-negative bacteria or $S$. saprophyticus) with $\geq 10^{2}$ colonies $/ \mathrm{mL}$ in nonvoided specimens

d. $\leq 10^{5}$ colonies $\mathrm{mL}$ of a single uropathogen (gram negative bacteria or $S$. saprophyticus) in a patient being treated with an effective antimicrobial agent for a urinary tract infection

e. physician diagnosis of a urinary tract infection

f. physician institutes appropriate therapy for a urinary tract infection 